\title{
Soft optical composites for tunable transmittance
}

\author{
F. López Jiménez ${ }^{\mathrm{c}}$, P. Upadhyaya ${ }^{\mathrm{a}}$, J. Liljenhjerte ${ }^{\mathrm{a}}$, P.M. Reis ${ }^{\mathrm{c}, \mathrm{b}}$, S. Kumar ${ }^{\mathrm{a}, *}$ \\ ${ }^{a}$ Institute Center for Microsystems (iMicro), Department of Mechanical and Materials Engineering, \\ Masdar Institute of Science and Technology, PO Box 54224, Abu Dhabi, UAE \\ ${ }^{b}$ Department of Mechanical Engineering, Massachusetts Institute of Technology, Cambridge, MA 02139 \\ ${ }^{c}$ Department of Civil \& Environmental Engineering, Massachusetts Institute of Technology, Cambridge, MA 02139
}

\begin{abstract}
We introduce a soft composite that is actuated mechanically to achieve switchable and tunable optical transmittance. Our design comprises a series of parallel opaque platelets embedded into an optically clear solicone-based elastomer matrix. Under an applied shear loading, the platelets rotate, thereby gradually increasing the transmittance of light through the device, in a controllable and reversible manner. Experiments on a prototype device are used to support finite element simulations that explore the parameter space of the system towards providing a set of design guidelines. Specifically, we study how the optical transmittance depends on the stiffness mismatch between the matrix and the platelets, as well as their initial orientation and aspect ratio. We also focus on the maximum attainable value of transmittance and the energetic requirements to achieve it.
\end{abstract}

Keywords: Soft materials, composites, transmittance, optical devices

\section{Introduction}

Lighting accounts for approximately $11 \%$ of the total U.S. electricity consumption [1]. Climate-adaptive smart windows that allow for dynamic actuation of daylight hold 5 great potential for energy savings $[2,3,4,5,6]$. Beyond architectural applications, light control systems are also relevant in aerospace [7], electronic displays [8], tunable focus lenses [9] and tunable apertures [10]. Active optical devices in these arenas that are available commercially

10 can be classified into three categories: electrochromic windows (ECWs) [11, 12, 13, 14, 15], polymer dispersed liquid crystals (PDLCs) $[16,17,7]$ and suspended particle devices (SPDs) $[18,19,20]$. One desirable feature for these systems is the ability to switch and tune the optical transmittance,

$15 \Phi$, defined as the fraction of incident light that is transmitted through a material, over a large range, in a fast and reversible manner. ECWs can be actuated with low switching voltages $(1-5 \mathrm{~V})$ and exhibit a moderate transmittance range, $\Delta \Phi=0.1-0.6$, but typically have slow switching 20 times $(10 \mathrm{~s})[14,15]$. PDLCs and SPDs are faster (switching times in the range $50-200 \mathrm{~ms}$ ). However, they require a constant power supply and have relatitively limited transmittance ranges: $\Delta \Phi=0.5-0.8$ and $\Delta \Phi=0.2-0.6$, respectively $[20,7]$. Exciting new directions for switch25 able optical devices involve using optically clear elastomers (e.g., polydimethylsiloxane, PDMS) with a precisely designed microstructure $[21,22,23]$, reinforcement with

\footnotetext{
* Corresponding author:S. Kumar

Email address: kshanmugam@masdar.ac.ae; s.kumar@eng.oxon.org (S. Kumar)
}

magnetically controlled inclusions [24, 25], silica nanoparticles [26] and paraffin composites [27], and PDMS-dye suspesions [28]. Nonetheless, fabrication strategies involving intricate small-scale architectures are technically challenging to implement, and the presence of inclusions can lead to failure through cavitation and nucleated rupture.

Here, we introduce a soft optical device that is actuated mechanically (simple shear) to yield fine control of the optical transmittance, over a wide range (Fig. 1). We shall refer to this device as an optical switch. Our design concept is similar to that of conventional window shutters but implemented into a contiguous material system, in a way that minimizes moving parts and is amenable to miniaturization (see schematic in Fig. 1a,b). The device comprises an optically clear elastomeric matrix, onto which we embed
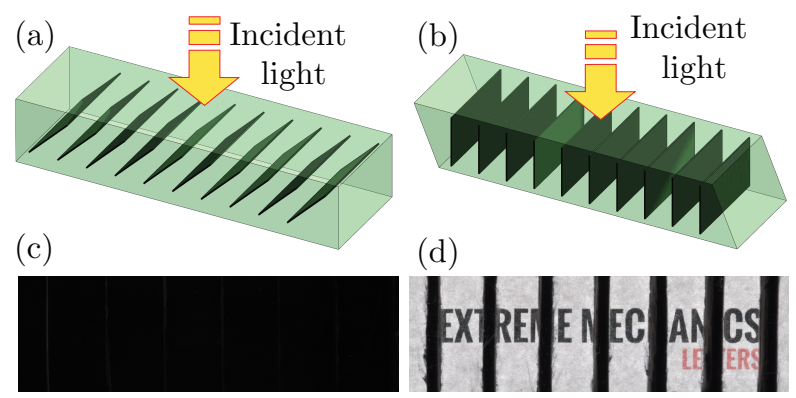

Figure 1: Concept of the optical switch: (a) Schematic representation of optical switch device in its undeformed (OFF) and (b) deformed (ON) configurations, respectively. (c) Photograph from experiments when the optical switch is in the OFF state, $\Phi=0$, and (d) after it has been toggled to is ON state, $0<\Phi<\Phi_{\max }$. 
a parallel array of thin opaque platelets that hinder light transmittance. Loading the device in simple shear forces 80 the initially inclined platelets (Fig. 1a,c) to follow the deformation field and rotate, such that the optical path becomes increasingly less obstructed and the transmittance increases (Fig. 1b,d). We perform experiments to both demonstrate a proof-of-concept of our design and to sup- 85 port finite element simulations that are then employed to systematically and quantitatively explore the parameter space. We find that the maximum transmittance depends strongly on the microstructure of the geometry (e.g., aspect ratio and initial orientation of the opaque platelets). 90 The stiffness mismatch has a small effect on the maximum optical transmittance, but it can be used to vary its evolution on the applied shear strain.

\section{Definition of the design concept}

In Fig. 2, we present a schematic diagram of our opti60 cal switch device. A block of transparent matrix (height $L_{y}$ in the direction of the incident light, and dimensions $L_{x}$ and $L_{z}$ in the other two directions) contains a peri-100 odic array of platelets (length $L_{p}$ and thickness $t_{p}$ ) with initial orientation $\theta_{i}$ with respect to the $x$-1axis. In our analysis we will distinguish between a central composite of matrix and platelets, with dimension $L_{c}=L_{p} \sin \theta_{i}$ in the $y$ direction, sandwiched between two regions of pure ${ }^{105}$ matrix, with combined size $L_{m}=L_{y}-L_{p} \sin \theta_{i}$ in the $y$ direction. The configuration shown in Fig. 2a corresponds

70 to the OFF state. To ensure full blockage of the light, the mid-point of the top edge of one platelet aligns with the mid-point of the bottom edge of its adjacent (marked as red dots in Fig. 2a), such that the distance between platelets is $d_{p}=L_{p} \cos \theta_{i}$. To facilitate actuation, the op-110 75 tical switch mechanism is sandwiched between and bonded to two rigid transparent plates. When an external shear strain $\gamma=U_{o} / L_{y}$ is applied, by imposing a displacement $U_{o}$ on the top plate of the device, the embedded platelets

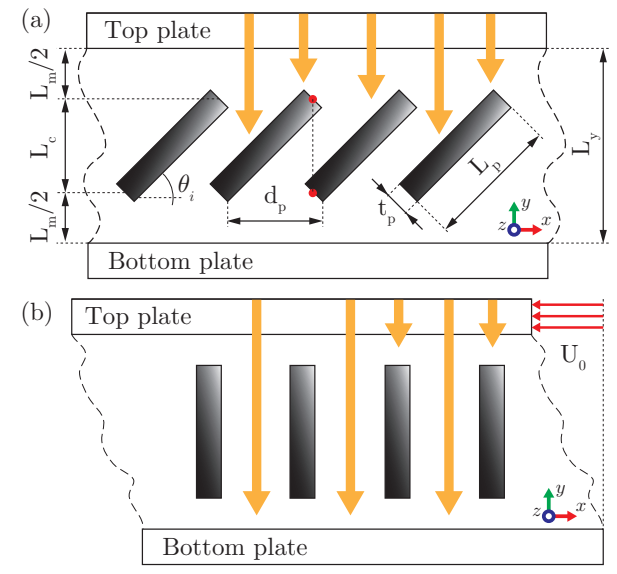

Figure 2: Schematic diagram of the optical switch, indicating all the relevant dimensions. (a) OFF (undeformed) state (b) ON (sheared) state. rotate and align with the incident light. We refer to this configuration as the ON state (Fig. 2b). The displacement of the plate in the $y$ and $z$ directions are constrained, so that $L_{x}$ and $L_{z}$ remain constant. The shear moduli of the matrix and the platelets are $\mu^{m}$ and $\mu^{p}$, respectively.

In this study we focus on the effect of the microstructure, i.e. the arrangement of platelets in the matrix, on the transmittance. Therefore, we assume that the platelets are completely opaque $\left(\Phi^{p}=0\right)$ and non-reflective, and that the matrix is perfectly transparent $\left(\Phi^{m}=1\right)$ in the visible light range. This is a reasonable assumption for the choice of materials in our experiments, detailed in $\S 4$, since PDMS is optically clear in the entire visible spectrum [28], and so we can assume that the transmittance is wavelength independent. The transmittance of the optical switch is therefore defined as the ratio of available 95 optical path for incident light perpendicular to the sample: $\Phi=1-A_{p} / A_{s w}$, where $A_{p}$ is the total projected area of the platelets on the top/bottom surface, and $A_{s w}$ is the total area of the top/bottom surface of the device. In the OFF state there is complete blockage of the light $(\Phi=0)$, requiring that $d_{p} \leq\left(L_{p}\left|\cos \theta_{i}\right|+t_{p}\left|\sin \theta_{i}\right|\right)$.

We proceed by exploring the parameter space of the system (geometry of the device and stiffness mismatch) through finite element simulations, with the goal of maximizing the optical transmittance, while minimizing the applied work. These simulations will subsequently be supported by experiments (in $\S 4$ ).

\section{Finite element simulations}

We have employed the Finite Element Method (FEM) using Abaqus/Standard to perform a geometrically nonlinear numerical analysis of the optical switch device presented in $\S 2$. The system was idealized as an infinite array of platelets embedded in the soft matrix. The constitutive behavior of both the matrix and platelets was assumed to follow an incompressible hyperelastic Neo-Hookean material model [29]. In order to reduce the computational cost, a series of preliminary studies with different Representative Volume Elements (RVEs) were performed. Regarding the $z$ direction, it was found that assuming plane strain overly constrained the system, since the platelets 20 can buckle when the stiffness mismatch is high, and therefore the full extent of $L_{z}$ had to be considered. Regarding the $x$-direction, periodic boundary conditions were used such that only a finite number of platelets needed to be considered. A parametric study showed that a single platelet with periodic boundary conditions provides the same results as that of larger RVEs. Nevertheless, four platelets were used throughout the study (three full platelets plus two half platelets, see Fig. 6c-e), in order to account for modes of deformation in which not all platelets rotated in sync. Additionally, this choice of RVE allows us to study the effect of small variations $\left( \pm 0.5^{\circ}\right)$ in the initial orientation of the platelets, in order to emulate fabrication imperfections, but this yielded negligible differences. 
The mesh for the RVE was constructed using eight-noded $3 \mathrm{D}$ hybrid brick elements $(\mathrm{C} 3 \mathrm{D} 8 \mathrm{H})$, with approximately 100,000 elements in the model.

The structure was sheared by fixing the bottom sur-180 face and applying a displacement $U_{0}$ on the top plate in the negative $x$ direction (see Fig. 2). The transmittance was determined from the $2 \mathrm{D}$ projection of the platelets onto the $x-z$ plane. The undeformed configuration had an initial obstructed projected area $A_{0}$, and the subsequent frames provided the aggregate instantaneous blocked area from all the platelets, $A_{i}$, which was then used to calculate transmittance as

$$
\Phi=1-\frac{A_{i}}{A_{0}}
$$

The same image processing procedure was followed to obtain the transmittance from the digitally acquired photographs in the experiments detailed next.

\section{Sample fabrication and experimental procedure}

To support the numerical simulations introduced above, we performed a series of experiments on an analogue elastomeric system. The samples were fabricated through casting, using PDMS (Sylgard 184, Dow Corning). First, 145 the mold was assembled using laser cut acrylic, including the top and bottom plates (eventually used to clamp and apply shear), ten equally spaced acrylic tabs (eventually removed and the void cast with PDMS to produce the platelets), and a set of side plates (required to fully enclose the volume). The base component of PDMS was mixed with its curing agent, first by hand for $5 \mathrm{~min}$, and then in a centrifugal mixer (ARE-310, Thinky) for $1 \mathrm{~min}$, to help degassing.

For the matrix, the ratio between curing agent and base was 1:62.5 (in weight), resulting in a shear modulus upon curing of $\mu^{m}=40 \mathrm{kPa}$ that was kept fixed for all experiments. The mixture was poured into the mold and degassed to remove air bubbles using a vacuum pump. Thereafter, the mold assembly was kept at $100^{\circ} \mathrm{C}$ for one hour to cure the elastomer. Next, the PDMS block was demolded from the side acrylic plates, except for the embedded acrylic tabs. Each of the ten tabs were then sequentially removed by sliding them out of the PDMS block, 185 performing the debonding carefully to prevent damage. Their respective voids were refilled with a new PDMS mixture containing black pigment in suspension (Silc-Pig, by Smooth-on). To ensure that the resulting platelets were completely opaque, a weight ratio of $\sim 0.5 \%$ was used be-190 tween the PDMS mixture and Silc-Pig. For the opaque platelets, a similar mixing procedure was followed to that of the matrix, except with different ratios of the base polymer to curing agent $(1: 50,1: 35,1: 30)$, to increase their relative stiffness to $\mu^{p} / \mu^{m}=3.6,28.9$, and 40.5 , respectively. A photograph of one of the fabricated samples is

175 shown in Fig. 3a,b. The elastic moduli of materials of ${ }^{195}$ both the matrix and the platelets were measured using additional samples of bulk material with the same mixing ratios stated above, and cured concurrently to the corresponding optical switch sample to ensure reliability of the measured quantities.

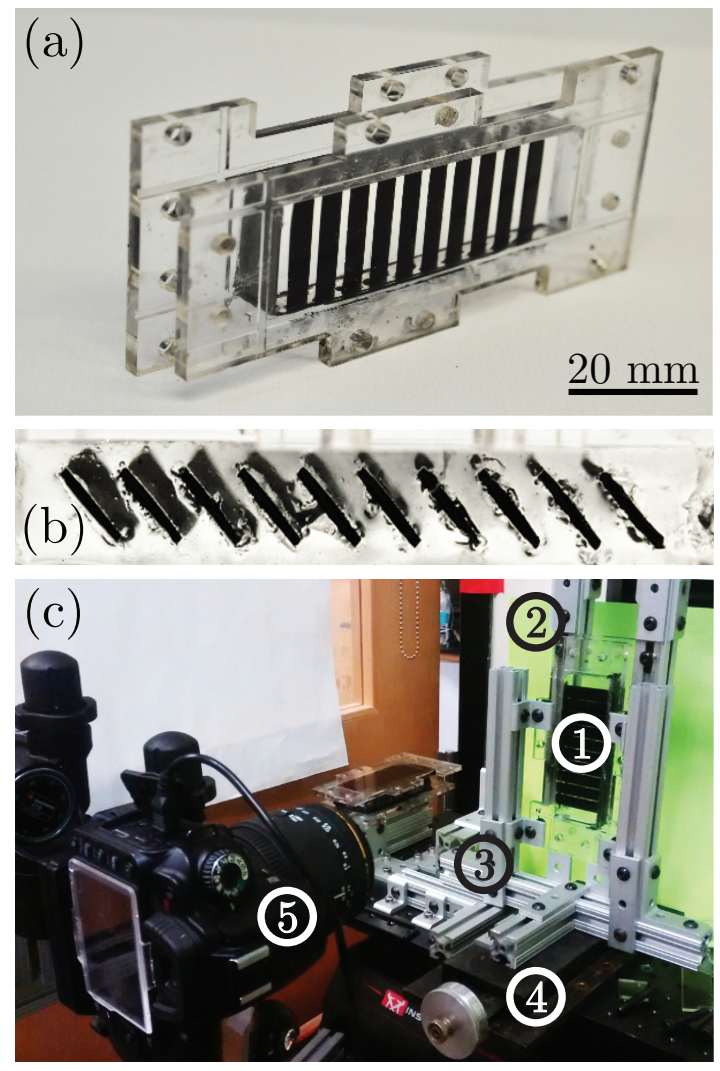

Figure 3: Experimental photographs. (a) Perspective and (b) top view of a specimen. (c) Photograph of the experimental apparatus. The sample (1) was attached to an Instron universal testing machine (2) using its back plate, and the front plate was mounted on the laboratory frame (3). A linear stage allowed for small adjustments in the $y$ direction for alignment. A digital camera (5) captured frames during experiments at regular intervals; to facilitate image processing a green background was used.

The specimens had ten platelets $\left(t_{p}=1.6 \mathrm{~mm}, L_{p}=\right.$ $14 \mathrm{~mm}$, and $\left.\theta_{i}=45^{\circ}\right)$, spaced regularly $\left(d_{p}=10 \mathrm{~mm}\right)$ in a matrix block with dimensions $\left(L_{x}, L_{y}, L_{z}\right)=(120,20,40) \mathrm{mm}$. These samples were tested using an universal testing machine (Instron 5943) and an uniform shear displacement was applied through top and bottom acrylic plates bound to the outer surfaces. A digital SLR camera (Nikon D90) placed perpendicularly to the setup acquired photographs of the projected area of platelets with a green background, see Fig. 3c. These images were post-processed to determine the transmittance, $\Phi$ [defined in Eq. (1)] as a function of the applied shear strain using the image-processing toolbox in MATLAB [30].

\section{Tunable transmittance: FEM and Experiments}

In Fig. 4a, we present a comparison between numerical and experimental results for the dependence of the transmittance, $\Phi$, as a function of the applied shear strain, $\gamma$, 

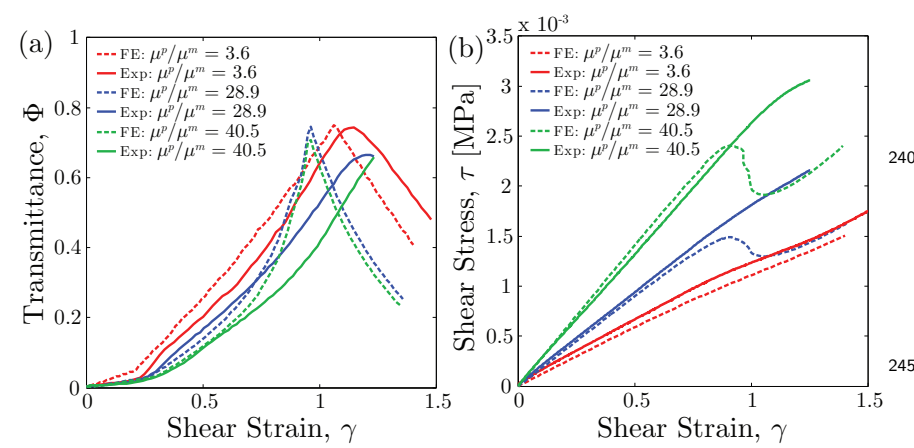

Figure 4: Comparison between FEM simulations (dashed lines) and experiments (solid lines) for three different values of the stiffness mismatch: $\mu^{p} / \mu^{m}=3.6,28.9,40.5$. (a) Optical transmittance, $\Phi$, as a function of the applied shear strain, $\gamma$. (b) Shear stress, $\tau$, as a function of the applied shear strain, $\gamma$.

for the three optical switch prototypes described in $\S 4$. Fig. $4 \mathrm{~b}$ shows the homogenized shear stress required for actuation. Focusing first on the numerical results (dashed lines), when the stiffness mismatch is low $\left(\mu^{p} / \mu^{m}=3.6\right)_{, 255}$ we find that the behavior of the device is close to that of an homogeneous block of PDMS; $\Phi$ and $\tau$ vary approximately linearly with the applied shear strain, $\gamma$, until the $\Phi_{\max }=0.75$ is reached. In the other two cases, the relative stiffness of the platelets is sufficiently high for ${ }_{260}$ them not to follow the same shear deformation as that of the matrix and, instead, undergo mostly rigid body rotation. As a result, their projected length in the $y$ direction creases and the region of the matrix above and below the platelets is compressed. In practice, this nonhomoge- ${ }_{265}$ neous stress state yields a nonlinear relationship between the applied shear strain and the rotation of the platelets, which in turn is responsible for the nonlinear behavior of seen in Fig. 4a. As the orientation of the platelets approaches that of the incident light $(\theta \rightarrow \pi / 2)$ the compressed portion of the matrix imparts a compressive load on the platelets which causes them to buckle, leading to the drops in shear stress seen in Fig. 4b. Despite this qual-

220 itatively different behavior between the cases of low and high stiffness ratio, the deviations on the maximum transmittance, $\Phi_{\max }$, between the three samples considered are of the order of $5 \%$.

The experimental results (solid lines in Fig. 4a,b) sup225 port the numerical results described above, especially for low values of the stiffness ratio $\left(\mu^{p} / \mu^{m}=3.6\right)$ for which the maximum transmittance $\Phi_{\max } \approx 0.74$ is in good agreement between the experiments and simulations. For higher values of the stiffness mismatch, and especially for large shear strains $(\gamma>0.75)$, the FEM simulations over-estimate the experimentally measured values of $\Phi$ (see Fig. 4a), with mismatches for the maximum transmittance of approximately $10 \%$ (together with an underestimation of the strain at which these values are reached). This discrepancies are attributed to debonding between the matrix and the plates, as well as small displacements in the $y$ direction.
Both of these artifacts lead to a relaxation of the effect of the incompressibility constraint and in turn alter the local stress distribution. Consequently, the experimentally measured shear force does not exhibit the sudden drop after the maximum load exhibited by the simulations (see Fig. 4b), presumably, because the platelets are allowed to rotate more freely without significant buckling deformation along the direction of their width. Despite the apparent discrepancies, it is important to note that there are no fitting parameters in the computation; all geometric and material parameters were measured independently.

\section{Exploring the design parameter space}

Thus far, we have demonstrated a proof-of-concept for 50 ulations qualitatively capture the overall optical and mechanical response of the system. We proceed by focusing on the numerics and conduct a systematic exploration of the parameter space towards the goal of identifying general guidelines to assist in future experiments and practical implementations of our concept. Following an initial broad exploration of parameters, we decided to give particular emphasis to the following parameters: the stiffness ratio, $\mu^{p} / \mu^{m}$, the initial angle of the platelets, $\theta_{i}$, and their aspect ratio, $L_{p} / t_{p}$. It was found that the relationship between $L_{m}$ and $L_{c}$ had little effect on the mechanical and no effect on the optical properties, besides altering the amount of global shear necessary to rotate the platelets. As such, $L_{m}=L_{c}$ was chosen as a representative value. The main objective of this effort is to gain insight into the conditions that may lead to an optimization of the performance of the device in terms of maximum achievable transmittance, $\Phi_{\max }$. In $\S 7$, below, we shall then quantify the external work required to achieve $\Phi_{\max }$.

First, we match the stiffnesses of the matrix and the platelets, $\mu^{p} / \mu^{m}=1$, and refer to this case as the $m e$ chanically homogeneous design. The initial angle and the aspect ratio of the platelets are varied in the ranges $15 \leq$ $\theta_{i}\left[^{\circ}\right] \leq 75$ and $5<L_{p} / t_{p}<80$, respectively, while keeping $L_{c}=L_{m}$, i.e. $L_{p}=L_{y} /\left(2 \sin \theta_{i}\right)$. The outer dimensions of the specimens are identical to those of the fabricated samples described in $\S 4$. In Figs. 5 a,b we plot the dependence of the transmittance $\Phi$ versus the shear strain $\gamma$, for different values of $L_{p} / t_{p}$ (see legends of the plots), while fixing $\theta_{i}=30^{\circ}$ and $\theta_{i}=75^{\circ}$, respectively. For low values of $\gamma$, the transmittance is zero, since the projection of the platelets on the $x$ direction overlaps initially by $t_{p} \sin \theta_{i} / 2$ (see Fig. 2a); a finite value of $\gamma$ is required to switch the device with $\Phi>0$. This threshold in $\gamma$ increases as the thickness of the platelets increases, such that the switching behavior is more pronounced for the lowest values of the platelet aspect ratio $\left(L_{p} / t_{p}\right)$. Past the switching onset, the transmittance increases linearly with applied shear strain, for all cases considered, reaching a clear maximum, $\Phi_{\max }$, at $\gamma_{\max } \approx 1 / \tan \theta_{i}$, after which $\Phi$ decreases linearly again. 

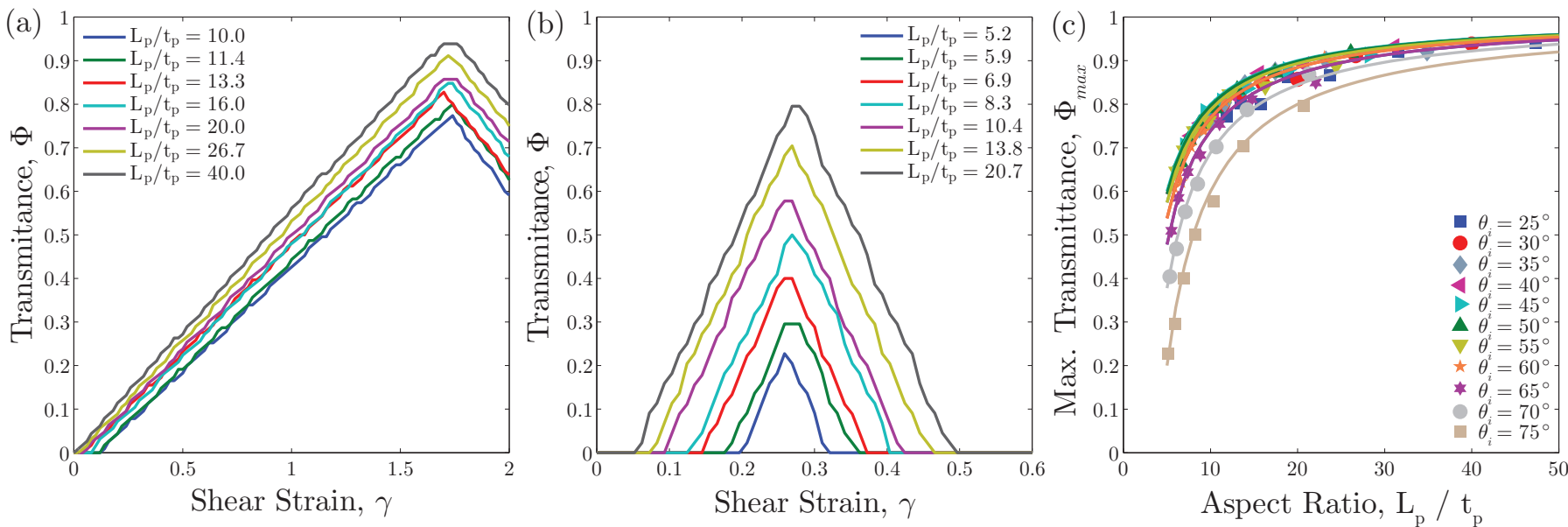

Figure 5: FEM simulations of the mechanically homogeneous design $\left(\mu^{p} / \mu^{m}=1\right)$ : Transmittance, $\Phi$ as a function of applied shear strain, $\gamma$ for different aspect ratios and initial platelet angle: (a) $\theta_{i}=30^{\circ}$ and (b) $\theta_{i}=75^{\circ}$. (c) Maximum transmittance, $\Phi_{\text {max }}$ versus the aspect ratio, $L_{p} / t_{p}$ for different initial angles. The data points correspond to FEM simulations, and the solid lines to the predictions in Eq. (2).

The value of $\Phi_{\max }$ is found to increase with the aspect ratio of the platelets, $L_{p} / t_{p}$, and decreases with the initial angle, $\theta_{i}$. This can be understood by noting that, as the platelets rotate to align with the $y$ direction in a matrix of the same stiffness, their length decreases to $L_{c}=L_{p} \sin \theta_{i \cdot 300}$ Since PDMS is nearly incompressible, their width expands to $t_{p} / \sin \theta_{i}$, ensuring volume conservation. The maximum transmittance can then be approximated to

$$
\Phi_{\max }=\frac{t_{p}}{\sin \theta_{i} \cos \theta_{i} L_{p}} .
$$

In Fig. 5c, we plot $\Phi_{\max }$ versus $L_{p} / t_{p}$, for different values of $\theta_{i}$ and find that the FEM data is in excellent agreement with the prediction from Eq. (2).

Next, we study the effect of the stiffness mismatch by considering the representative values $\mu^{p} / \mu^{m}=\{10,20$, $50,100$ and 1000$\}$, while fixing $\theta_{i}=55^{\circ}$ and $L_{p} / t_{p}=24.4$. Fig. 6 presents the transmittance as a function of the applied shear deformation. We find that as $\mu^{p} / \mu^{m}$ is increased, the $\Phi(\gamma)$ data becomes increasingly more nonlinear. This finding can be rationalized by recognizing that during the rotation of the platelets under shear loading (to align them with the direction of the incident light), they are required to shorten and are forced into a state of compression. For low values of $\mu^{p} / \mu^{m}$ (e.g., $\mu^{p} / \mu^{m}=1$ and 10; see the FEM snapshots of Fig. 6c,d), this compression is accommodated by the compliance of the platelets, which are mostly advected by the matrix. By contrast, for higher values of $\mu^{p} / \mu^{m}$, the strain response of the system becomes inhomogeneous with localization occurring in regions of the matrix away from the platelets. Interestingly, for $\mu^{p} / \mu^{m} \geq 50$, this compression leads to buckling of the platelets (see FEM snapshot in Fig. 6e for $\mu^{p} / \mu^{m}=100$ ), which is responsible for the maxima in the corresponding shear stress-strain curves, $\tau(\gamma)$, presented in Fig. $6 \mathrm{~b}$. Note that over this wide range of $\mu^{p} / \mu^{m}$, the maximum transmittance attained remained approximately constant at $\Phi_{\max } \approx 0.88$, with a reduction of at most $\sim 10 \%$ when the platelets undergo significant buckling $\left(e . g ., \mu^{p} / \mu^{m}=\right.$ 50 and 100). If the stiffness mismatch is increased even further, $\mu^{p} / \mu^{m}=1000$, the buckling is greatly reduced, due to the stiffness of the platelets, and so the maximum transmittance is again $\Phi_{\max } \approx 0.88$. In this case, the drop in shear loading is associated with the increase in shear deformation in the matrix, required to allow the platelets to rotate without shortening. Stiffer platelets also lead to a higher value of the overall shear stiffness of the device, the initial slope in the $\tau(\gamma)$ curves in Fig. $6 \mathrm{~b}$, but only by at most a factor of 1.5 , even for $\mu^{p} / \mu^{m}=1000$. The reason is that the shear response of a composite is dominated by
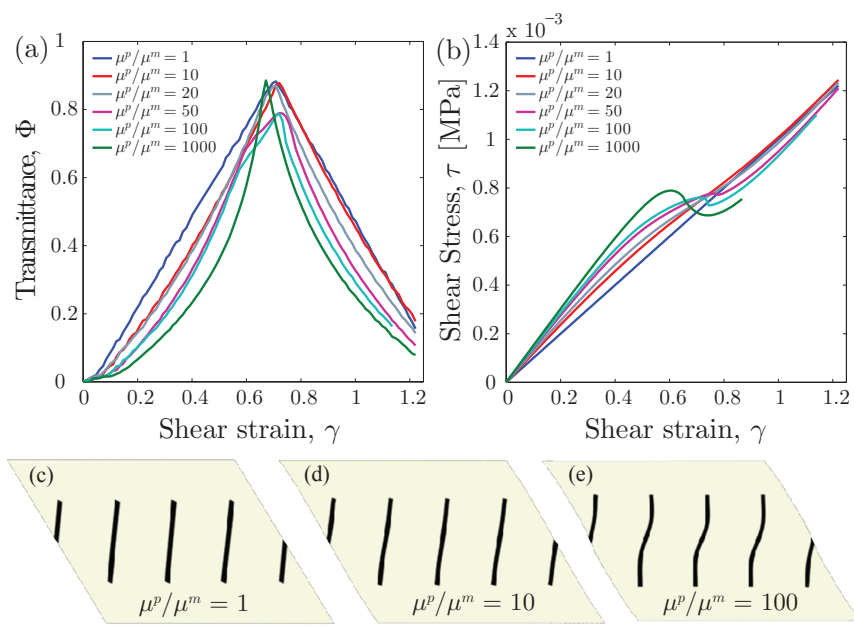

Figure 6: FEM simulations of the mechanically inhomogeneous design: (a) Transmittance $\Phi$ and (b) shear stress $\tau$ as functions of the applied shear strain, $\gamma$, for values of stiffness ratio in the range $1 \leq \mu^{p} / \mu^{m} \leq 1000$. Side view of deformed samples at $\gamma=0.7$ for different stiffness ratios (c) $\mu^{p} / \mu^{m}=1$ (d) $\mu^{p} / \mu^{m}=10$ (e) $\mu^{p} / \mu^{m}=100$. The initial angle $\theta_{i}=55^{\circ}$ and aspect ratio $L_{p} / t_{p}=24.4$ for all cases. 
the properties of the matrix [31], with a weak dependance 335 on the properties of the platelets (reinforcement).

The parametric study presented above identifies the aspect ratio of the platelets as the most critical parameter in dictating the full range of transmittance that the device is able to span. On the other hand, the effect on ${ }_{340}$ the initial angle of the platelets is less pronounced, especially for designs with high values of $L_{p} / t_{p}$. The maximum range of optical transmittance was found for $L_{p} / t_{p}>40$ $\left(\Phi_{\max } \approx 0.95\right)$ but we note that such slender platelets may pose implementation challenges in their fabrication.345 all the other designs that we have explored offer $\Phi_{\max }>0.8$, which is already comparable or superior to commercially available technologies [2]. Finally, varying the stiffness ratio between the matrix and the platelets makes it possible to modulate the extent of nonlinearity of 350 the $\Phi(\gamma)$ response.

\section{External work for maximum transmittance}

Having characterized the optical and mechanical re-355 sponse of our optical switch device as a function of the various parameters of the system, we now quantify the external work per unit area (in the $y-z$ plane), $W_{M T}$, required to achieve the configuration that yields maximum transmittance. To do so, we integrate the area under the ${ }_{360}$ stress-strain curves, from the undeformed state $\gamma_{0}$ (corresponding to $\Phi=0$ ), up to the state of maximum transmittance $\gamma_{\max }$ (corresponding to $\left.\Phi=\Phi_{\max }\right)$ :

$$
W_{M T}=L_{y} \int_{\gamma_{0}}^{\gamma_{\max }} \tau d \gamma
$$

We now define $\eta=W_{\mathrm{MT}} / W_{0}$ as the dimensionless external work in Eq. (3), normalized by the work, $W_{0}$, required to achieve unit shear strain of a cuboid of bulk PDMS with identical outer dimensions as the specimen and stiffness of the matrix.

We first consider the mechanically homogeneous de$\operatorname{sign}\left(\mu^{p} / \mu^{m}=1\right)$. In Fig. 7a, we plot $\eta$ as a function of $L_{p} / t_{p}$, for different values of the initial angle of the platelets. The data shows no visible dependence on the aspect ratio, since both the platelets and the matrix have the same stiffness, while the values of $\eta$ increase significantly as $\theta_{i}$ is decreased. This finding can be better understood by considering that the shear strain necessary to align the platelets with the direction of incident light, and therefore achieve maximum transmittance, can be approximated solely as a function of the initial platelet angle, $\gamma_{\max }=\cot \theta_{i}$, such that, if the shear modulus is assumed to be constant, one arrives at:

$$
\eta=\frac{L_{y} \int_{\gamma_{0}}^{\cot \theta_{i}} \mu^{m} \gamma d \gamma}{L_{y} \int_{\gamma_{0}}^{1} \mu^{m} \gamma d \gamma}=\cot ^{2} \theta_{i}
$$

We now consider cases for which the platelets are stiffer than the matrix. In Fig. 7b, we plot the dimensionless external work, $\eta$, for a device with $\mu^{p} / \mu^{m}=20$, as a function of the platelet aspect ratio, for a variety of initial platelet angles. The work decreases for higher values of platelet aspect ratio, since as $L_{c}$ is kept constant, these cases correspond to a lower volume fraction of the reinforcement, and, hence, a reduced shear stiffness of the device. The effect of the initial angle $\theta_{i}$ on the external work is similar to the homogeneous case: lower values of $\theta_{i}$ require higher values of applied shear to achieve maximum transmittance, and so the external applied work is also higher.

In Fig. 7c, the dimensionless external work $\eta$ is now plotted versus the stiffness ratio, for different aspect ratios, at the fixed value of initial angle, $\theta_{i}=55^{\circ}$. Although increasing the stiffness of the platelets increases the required applied work, the effect is relatively small, and $\eta$ eventually attains a plateau. This finding can be rationalized by the fact that the shear response of a composite is dominated primarily by the matrix, and so the effect of increasing the stiffness of the reinforcement (platelets) in our system is small.

In order to provide an analytical prediction for $\eta$ in the nonhomogeneous case $\left(\mu^{p} / \mu^{m}>1\right)$, we idealize the switch device as a combination of three regions: a composite of platelets and matrix, of size $L_{c}$ in the $y$-direction, sandwiched between two identical regions of pure matrix, each of them of size $L_{m} / 2$ in the $y$ direction. Due to difference in stiffness between the composite and the pure matrix, their respective shear strains, $\gamma^{c}$ and $\gamma^{m}$, are also different. We start our analysis by focusing on the central region, defined as a composite of platelets and matrix. The volume fraction of each component can be approximated from the geometry of the specimen as:

$$
\begin{aligned}
V_{p} & =\frac{t_{p}}{L_{p} \sin \theta_{i} \cos \theta_{i}}, \\
V_{m} & =1-V_{p} .
\end{aligned}
$$

The mechanical properties of the composite region can be calculated using estimates for the micromechanics of fiber composites [31], as a function of the properties of the components. Defining a material frame $\mathbf{e}_{1}-\mathbf{e}_{2}$, where $\mathbf{e}_{1}$ is parallel to the fiber (platelets) direction, the homogenized stiffness in the $\mathbf{e}_{1}$ and $\mathbf{e}_{2}$ directions $\left(E_{1}\right.$ and $E_{2}$, respectively) and the in-plane Poisson's ratio and shear stiffness $\left(\nu_{12}\right.$ and $\left.\mu_{12}\right)$ are given by:

$$
\begin{aligned}
E_{1}^{c} & =V_{p} E_{p}+V_{m} E_{m}, \\
E_{2}^{c} & =\frac{E_{m} E_{p}}{V_{m} E_{m}+V_{p} E_{p}}, \\
\nu_{12}^{c} & =V_{p} \nu^{p}+V_{m} \nu^{m} \\
\mu_{12}^{c} & =\frac{\mu^{m}}{V_{m}+V_{p}\left(\mu^{m} / \mu^{p}\right)},
\end{aligned}
$$

The shear stiffness in the global coordinates $x-y, \mu_{x y}$, is a function of the material-frame properties of the composite, as well as the current orientation of the platelets, $\theta$, which is the angle between the $x$ and the $\mathbf{e}_{1}$ axis. It can be 

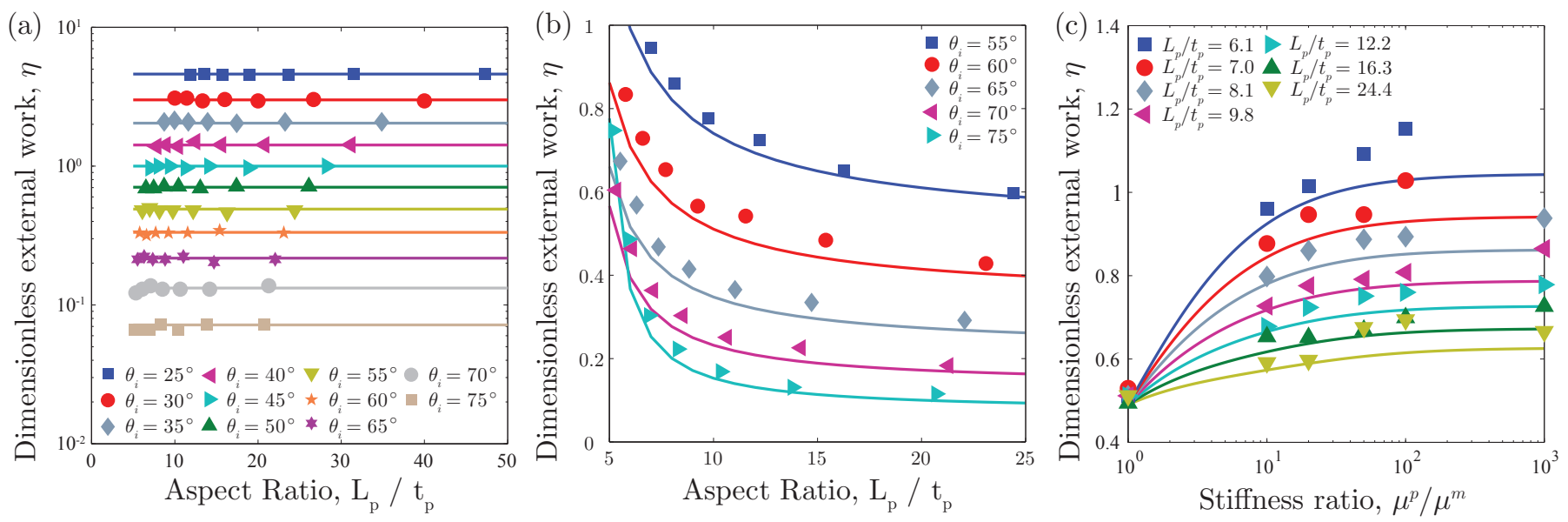

Figure 7: (a) External work required to reach maximum transmittance $\Phi_{\max }$ as a function of the aspect ratio, $L_{p} / t_{p}$ for different initial angles in a homogeneous design $\left(\mu^{p} \mu^{m}=1\right)$. (b) External work required to reach maximum transmittance $\Phi_{\max }$ as a function of aspect ratio $L_{p} / t_{p}$ for different initial platelet angles $\theta_{i}$ and stiffness mismatch $\mu^{p} / \mu^{m}=20$. (c) External work required to reach maximum transmittance $\Phi_{\max }$, as a function of the stiffness mismatch $\mu^{p} / \mu^{m}$, for different values of the platelet aspect ratio, $L_{p} / t_{p}$ and initial angle $\theta_{i}=55^{\circ}$. The data points correspond to FEM simulation results, and the solid lines to the prediction in (a) Eq. (4) and (b-c) Eq. (13).

expressed as:

$$
\begin{aligned}
\frac{1}{\mu_{x y}^{c}}= & 2\left(\frac{2}{E_{1}^{c}}+\frac{2}{E_{2}^{c}}+\frac{4 \nu_{12}^{c}}{E_{1}^{c}}-\frac{1}{\mu_{12}^{c}}\right) \sin ^{2} \theta \cos ^{2} \theta \\
& +\frac{1}{\mu_{12}^{c}}\left(\sin ^{4} \theta+\cos ^{4} \theta\right)
\end{aligned}
$$

Assuming that the structure is loaded in pure shear, and that the shear stress is constant across both regions, the shear strain in the matrix region, $\gamma^{m}$, can be calculated ${ }^{390}$ as a function of the shear in the composite region, $\gamma^{c}$, as:

$$
\gamma^{m}=\gamma^{c} \frac{\mu_{x y}^{c}}{\mu^{m}}
$$

Making the additional assumption that the maximum transmittance is achieved when the shear strain in the composite region is $\gamma_{\max }^{c}=\cot \theta_{i}$, the required mechanical work can be calculated as:

$$
\eta=\frac{2}{L_{y} \gamma^{m}}\left(L_{m} \int_{0}^{\gamma_{\max }^{m}} \mu^{m} \gamma^{m} d \gamma^{m}+L_{c} \int_{0}^{\gamma_{\max }^{c}} \mu^{c} \gamma^{c} d \gamma^{c}\right),
$$

where the integrals need to take into account the fact that $\mu_{x y}^{c}$ depends on the current angle of the platelets with respect to the $x$ direction, $\theta$, which can be calculated using the relationship

$$
\gamma^{c}=\cot \theta_{i}-\cot \theta
$$

Fig. $7 \mathrm{~b}, \mathrm{c}$ show the predictions obtained by numerical integration of Eq. (13). They provide good agreement with the FEM results and can be used as guidelines in future ${ }_{410}$ designs. The disagreement between FEM and analytical prediction becomes more important for high values of the stiffness mismatch, when the simplifications of this homogenization approach (e.g., neglecting the material nonlinearities of PDMS and the stress in the $y$ direction due to 415 the rotation of the platelets) become more significant, see Fig. 7c.

Overall, these results show that the work required to reach the maximum optical transmittance of our optical switch device decreases as both the initial angle, $\theta_{i}$, and aspect ratio, $L_{p} / t_{p}$, of the platelets are increased. The effect of the stiffness ratio between matrix and platelets is significantly smaller, due to the fact that the total shear response is dominated by the matrix.

\section{Discussion and Conclusions}

We have introduced a novel design for a soft optical composite that can be used to switch and tune optical transmittance, on demand. Our device is composed of a transparent elastomeric matrix (PDMS) reinforced with a series of parallel opaque platelets, which rotate when the system is actuated mechanically through simple shear. This mechanism is fully reversible and allows a controllable and incremental variation in optical transmittance. We presented experimental results that provided a proofof-concept of our design. A systematic exploration of parameter space using FEM simulations, together with a reduced analytical model, were then performed to quantify the optical performance, mechanical response and actuation work requirements of our device. We found that high optical transmittance, $\Phi_{\max }$, and low dimensionless external work required for actuation, $\eta$, can be achieved through a design that combines low values of the stiffness ratio with high values of both the initial angle of the platelets and their aspect ratio.

We further contextualize our design for an optical switch by comparing its actuation requirements with typical values found in the literature for other technologies. For mechanically homogeneous designs with different initial angles, $15^{\circ}<\theta_{i}<75^{\circ}$, and considering only cases that 
achieve high maximum transmittance, $\Phi_{\max }>0.7$, at moderate shear strains, $\gamma\left(\Phi=\Phi_{\max }\right)<1.5$, we found ${ }^{475}$ that the mechanical work required to actuate our optical switch is in the range $0.0087<W_{\mathrm{MT}}\left[\mathrm{Wh} / \mathrm{m}^{2}\right]<0.24$. to actuate ECWs, the most energy economical commer-480 cial smart windows, reported as $0.04 \mathrm{Wh} / \mathrm{m}^{2}$ [2] in the literature.

Throughout this study, we have focused on a design with zero transmittance in the default configuration, and ${ }^{48}$ maximum transmittance under shear loading. This could be easily modified by choosing a different initial angle for the platelets, so that they would align, at rest, with the direction of the incident light. As a result, shearing would re- ${ }^{490}$ duce the transmittance. In fact, there are possible applications for our device, such as architecture, where variations in the light direction are expected, and so the shear strain required to achieve maximum and minimum transmittance ${ }^{495}$ would not be constant. In that case, it may be advantageous to choose an initial orientation of the platelets that minimizes the maximum applied force for the expected range of incident angles. This alternative design is left for ${ }^{500}$ a more detailed analysis in a future study.

Even though our centimetric specimens were fabricated for proof-of-concept, the simplicity of our design and its purely mechanical operating principle open the potential ${ }^{505}$ for scalability in fabrication and actuation. Moreover, the exceptional mechanical properties of PDMS and other optically clear elastomers make this design mechanically robust and adaptable to a variety of geometries and working conditions. We therefore hope that devices for tunable transmittance based on our design will find applications not only in architecture, but also in other fields where active optical actuation is required, such as microfluidics or ${ }^{515}$ photolithography.

Acknowledgments: This work was supported by the Cooperative Agreement between the Masdar Institute of Science and Technology (Masdar Institute), Abu Dhabi, UAE and the Massachusetts Institute of Technology (MIT), Cambridge, MA, USA (Reference 02/MIT/MI/CP/11/07633/GEN/G/00) and by the National Science Foundation (CAREER CMMI-1351449).

\section{References}

[1] The U.S. Energy Information Administration (EIA), 2010 U.S. Lighting Market Characterization, http: //apps1.eere.energy.gov/buildings/publications/pdfs/ ssl/2010-lmc-final-jan-2012.pdf, 2012. [Online; accessed 20-July-2015]

[2] R. Baetens, B. P. Jelle, A. Gustavsen, Sol. Energ. Mat. Sol. Cells 94 (2010) 87-105.

[3] J. Backholm, E. Avendaño, A. Azens, G. de M Azevedo, E. Coronel, G. A. Niklasson, C.-G. Granqvist, Sol. Energ. Mat. Sol. Cells 92 (2008) 91-96.

[4] K. Bange, Sol. Energ. Mat. Sol. Cells 58 (1999) 1-131.

[5] S. Bao, Y. Yamada, M. Okada, K. Yoshimura, Appl. Surf. Sci. 253 (2007) 6268-6272

[6] W. Gao, S. Lee, J. Bullock, Y. Xu, D. Benson, S. Morrison, H. Branz, Sol. Energ. Mat. Sol. Cells 59 (1999) 243-254.
[7] C. M. Lampert, Sol. Energ. Mat. Sol. Cells 76 (2003) 489-499.

[8] R. A. Hayes, B. Feenstra, Nature 425 (2003) 383-385.

[9] S. Xu, Y. Liu, H. Ren, S.-T. Wu, Opt. Express 18 (2010) 1243012435.

[10] C. Liu, Q.-H. Wang, M.-H. Wang, Optik 125 (2014) 6661-6664.

[11] G. Bodurov, P. Stefchev, T. Ivanova, K. Gesheva, Mater. Lett. 117 (2014) 270-272.

[12] Z. Bi, S. Zhang, X. Xu, X. Hu, X. Li, X. Gao, Mater. Lett. 160 (2015) 186-189.

[13] C. G. Granqvist, Thin Solid Films 564 (2014) 1-38.

[14] S. K. Deb, S.-H. Lee, C. E. Tracy, J. R. Pitts, B. A. Gregg, H. M. Branz, Electrochimica Acta 46 (2001) 2125-2130.

[15] C. Xu, L. Liu, S. E. Legenski, D. Ning, M. Taya, J. Mater. Res. 19 (2004) 2072-2080.

[16] H. H. Khaligh, K. Liew, Y. Han, N. M. Abukhdeir, I. A. Goldthorpe, Sol. Energ. Mat. Sol. 132 (2015) 337-341.

[17] Y. Kim, D. Jung, S. Jeong, K. Kim, W. Choi, Y. Seo, Curr. Appl. Phys. 15 (2015) 292-297.

18] S. Leinberg, V. Kisand, A. Šutka, K. Saal, R. Lõhmus, U. Joost, M. Timusk, E. Nõmmiste, Opt. Mater. 46 (2015) 418-422.

[19] K. Moradi, B. El-Zahab, Opt. Mater. 47 (2015) 582-585.

[20] R. Vergaz, J.-M. Sánchez-Pena, D. Barrios, C. Vázquez, P. Contreras-Lallana, Sol. Energ. Mat. Sol. Cells 92 (2008) $1483-1487$.

[21] S. G. Lee, D. Y. Lee, H. S. Lim, D. H. Lee, S. Lee, K. Cho, Adv. Mater. 22 (2010) 5013-5017.

[22] E. Lee, M. Zhang, Y. Cho, Y. Cui, J. Van der Spiegel, N. Engheta, S. Yang, Adv. Mater. 26 (2014) 4127-4133.

[23] D. Park, P. Kim, J. Alvarenga, K. Jin, J. Aizenberg, M. Bechthold, Build. Environ. 82 (2014) 87-96.

[24] Y. Zhu, D. Antao, R. Xiao, E. Wang, Adv. Mater. 26 (2014) 6442-6446.

[25] A. Rotzetter, R. Fuhrer, R. Grass, C. Schumacher, P. Stoessel, W. Stark, Adv. Eng. Mat. 16 (2014) 878-883.

[26] D. Ge, E. Lee, L. Yang, Y. Cho, M. Li, D. S. Gianola, S. Yang, Adv. Mater. 27 (2015) 2489-2495.

[27] H. Apostoleris, M. Chiesa, M. Stefancich, J. Mater. Chem. C 3 (2015) 1371-1377.

[28] F. López Jiménez, S. Kumar, P. Reis, Adv. Opt. Mater. (2016).

[29] R. Ogden, Non-linear elastic deformations, Courier Corporation, 1997.

[30] MATLAB, version 7.14.0.379 (R2012a), The MathWorks Inc., Natick, Massachusetts, 2012.

[31] R. Jones, Mechanics of composite materials, volume 193, Scripta Book Company Washington, DC, 1975. 